\title{
On motion periodicity of dynamic textures
}

\author{
Dmitry Chetverikov and Sándor Fazekas \\ Computer and Automation Research Institute \\ Budapest, Hungary \\ csetverikov@sztaki.hu
}

\begin{abstract}
Dynamic textures (DTs) are videos of natural or artificial processes, such as waves, smoke, fire, or walking crowd. While activities and motion events produced by moving shapes are well localised in space and time, the spatiotemporal extent of most natural DTs is less definite. The notion of periodicity, or regularity, has been extensively studied in static texture analysis and in activity analysis, where it proved very useful. At the same time, much less attention has been paid to temporal (quasi-) periodicity of dynamic textures, despite the obvious fact that this property is inherent in dynamic texture. We discuss the reasons, then present a framework for quantitative motion periodicity analysis of DTs. Using the optic flow and adapting the SVD-based algorithm for signal period estimation [17], we measure degree of periodicity of natural dynamic textures. Numerous test results are presented, including the application of the temporal periodicity features to DT classification.
\end{abstract}

\section{Introduction}

Following the terminology of the pioneering paper [20], three visual motion pattern categories are usually distinguished, namely, motion events, activities, and dynamic (temporal) textures. Individual motion events, like a single opening of a door, possess no temporal or spatial periodicity; they are beyond the scope of this study which is devoted to periodicity analysis. Activities of moving non-rigid objects, like digging, are periodic visual motion patterns localised in space and time. Finally, dynamic textures are spatially repetitive, time-varying motion sequences exhibiting certain temporal stationarity. This stationarity, which assumes temporal repetitiveness, is a basis of most dynamic texture models (e.g., [7]) and methods for DT recognition [6].

Periodicity analysis, which has a long history in applied signal processing [17], has also been fruitful in computer vision. Spatial texture synthesis and recognition $[18,5,10$, 9] and activity detection, segmentation and behaviour analysis (gait, running, jumping, etc.) $[24,2,8]$ are probably the best known examples of its application. As pointed out in the studies [5, 24], periodicity facilitates learning of the repeating regular pattern, extrapolating the pattern, or detecting irregularities, or abnormal events, within regular data. For learning, accurate detection and alignment of the repeating pattern $[24,9]$ and estimation of the period length are necessary; measuring the degree of periodicity is not needed.

This latter circumstance may be one of the reasons why temporal periodicity analysis of dynamic texture has received little attention. Although it is generally recognised that periodicity is inherent in dynamic texture, and it can probably serve as a useful feature 
for DT description and recognition, very few attempts to explicitely measure this property are known. The recent survey [6] mentions just a few methods that keep track of DT 'history' to a certain, limited temporal depth [3, 11, 21, 22]. With small depth, one can only estimate short-term temporal variations such as acceleration [19]; no periodicity estimation is possible. Global spatiotemporal transforms like spatiotemporal wavelets [25] may become useful in multiscale periodicity analysis when motion periodicity appears at different scales (e.g., trunk, branches, leaves of a tree in the wind). However, such transform should be applied to a large spatiotemporal volume, which has not been done yet.

We are motivated by the challenges of measuring temporal periodicity in dynamic texture: (1) lack on vision algorithms to start/compare with; (2) large volume of the spatiotemporal data to process; (3) non-stationarity of the signals involved; (4) need to measure noisy, low-periodicity signals; (5) need to provide a periodicity measure that behaves consistently and contributes to recognition of real-world DTs.

These challenges cannot be addressed by the methods used in activity recognition; in particular, alignment of the repeating pattern [24,9] and spatiotemporal salient points [8] are not applicable to smoke, fire, shower, or others DT not composed of well-detectable moving shapes. As we discuss later, Fourier Transform is not a good solution either.

The contributions of this paper are as follows. Using optic flow, we adapt the SVDbased algorithm [17] to periodicity estimation, which allows us to measure the degree of periodicity of natural dynamic textures. In doing so, we (1) provide a signal preprocessing procedure that renders the method applicable to a noisy signal riding on a nonlinear trend; (2) introduce different measures and analyse their behaviour at growing noise level; (3) address the issue of periodicity variation within a signal (non-stationarity); (4) compare the SVD-based method to other methods, such as signal minima and zerocrossing analysis; and, finally, (5) apply the introduced temporal periodicity features to recognition of 26 dynamic textures.

\section{DT analysis with optic flow}

By estimating motion periodicity, we hope to obtain a richer description of dynamic texture and contribute to the solution of different problems related to DT processing and recognition. Beyond the standard tasks of texture analysis for static and dynamic data, such as classification and segmentation, one can also aim at finding a (known or unknown) texture in an image, or dynamic texture in a video. The complementary problem is to find a defect in a texture, or an irregular event in a DT. An example of the latter is searching for an unusual behaviour in a walking crowd. When no prior information is available on the expected properties of the irregularities, this task is essentially outlier detection with learning on the regular majority data. Finally, DT separation, that is, separation of partially transparent, overlapping dynamic textures seems to have no analogue on the static side, since this is mainly possible due to motion.

As emphasised in the survey [6], the optic flow based approaches are currently by far the most popular among different approaches to DT description and recognition. We also use optic flow that allows us to concentrate on the dynamic component of a DT, which is of primary importance for temporal periodicity analysis. The appearance component is less important for us; this component can be added when desired, for example, to use colour and shape for better segmentation. In the current study, we intentionally ignore the 
appearance component in order to understand how much information visual motion can provide about the classes of natural and artificial processes captured by videos.

A selection of fast and precise optic flow algorithms is available, ranging from the simple normal flow to the modern implementation [16] of the classic Horn-Schunk procedure [15] and the method [4] that uses more sophisticated spatiotemporal smoothness constraints, as well its extension [1] that applies pre-segmentation to better respect motion discontinuities. As demonstrated in the study [12], the choice of optic flow algorithm is a critical issue when temporal textures are processed. It is more critical here than in the case of large moving objects because of numerous fine details in motion and more frequent discontinuities and occlusions.

A related important issue is that of measuring the confidence of the optic flow values. In the studies $[19,12]$, the confidence measure proposed in [14] was used to weigh the contribution of a pixel to an optic flow statistics. It was demonstrated that the weighting results in better classification rates, which can be attributed to an efficient removal of flow outliers as well as the pixels where the flow cannot be calculated due to the lack of motion information. In particular, the confidence measure [14] is composed of the eigenvalues of the spatiotemporal structure tensor and reflects its rank. The rank should be two for unambiguous calculation of flow; other values mean no apparent motion, the aperture problem, or flickering.

In this study, we test several algorithms for optic flow and two different confidence measures. Besides the described one, we also test a new confidence measure that will be presented in detail in a forthcoming paper. The idea is to roughly pre-segment the image based on the warping error between frames $F_{k}$ and $F_{k+1}$ : we warp back pixels in $F_{k+1}$ by the flow, then calculate the difference between warped values and $F_{k}$. The warping error is an indicator of a dynamic texture, with its frequent discontinuities and occlusions.

\section{Periodicity estimation}

In the rest of the paper, we will assume that an accurate optic flow has been calculated using the above mentioned methods, and the average flow magnitude in each frame has been computed as a function of time, or frame index, resulting in a one-dimensional temporal signal. (We will consider other flow features in future research.) From now on, we will deal with such signals and speak of signals rather than images.

In this section, we present several algorithms for signal periodicity estimation. The core of the section is the method by Kanjilal, Bhattacharya and Saha [17] which we adapt to our needs. Originally, this method was designed for period length estimation of quasiperiodic time-varying data such as medical signals (ECG, EEG), global temperature time series, solar activity, electrical power load pattern in urban areas, and many others. For us, the period length is less important, as we focus on periodicity measurement. We will demonstrate that the method [17] can be used for this purpose as well.

To better illustrate our goals, let us jump for a moment to some numerical results of the algorithm we are going to present now. Imagine we are generating a sequence of digital signals with growing Gaussian noise added to the amplitude, as shown in figure 1. The initial signal is periodic with the period length 25 . When $\sigma_{\text {noise }}=1.0$, one can hardly recognise any periodicity in the deteriorated signal. Still, we expect the method to measure the periodicity and the period, and it actually does: figure 2 demonstrates that this is 
possible. Below, we explain how the basic SVD-based method works.

\subsection{The basic SVD method}

As discussed in [17], a (nearly) periodic signal has three periodicity attributes: (1) period length; (2) pattern over successive repetitive segments; and (3) scaling factors associated with repetitive segments. Note that a periodic component embedded in a signal may not be sinusoidal and may contain segments that scale differently.

Using the notation of [17], consider a digital signal $x(k)$. Assume that the period is $n$ and place the successive $n$-intervals of $x(k)$ into the rows of the $m \times n$ data matrix

$$
A_{n}=\left[\begin{array}{cccc}
x(1) & x(2) & \ldots & x(n) \\
x(n+1) & x(n+2) & \ldots & x(2 n) \\
\ldots & \ldots & \ldots & \ldots \\
x((m-1) n+1) & \ldots & \ldots & x(m n)
\end{array}\right]
$$

If $n$ equals the period length, the rows of $A_{n}$ are linearly dependent, even when the scaling factors of the rows are different. The method uses the robust Singular Value Decomposition (SVD) to test the 'rank oneness' of the matrix $A_{n}$ and determine the repeating pattern and the scaling factors.

Applying SVD, we decompose the data matrix as $A_{n}=U S V^{T}$, where $U U^{T}=U^{T} U=$ $I, V V^{T}=V^{T} V=I$. The diagonal matrix $S$ contains the sorted singular values: $S=$ $\operatorname{diagonal}\left(s_{1}, s_{2}, \ldots, s_{r}: 0\right), s_{1} \geq s_{2} \geq \ldots s_{r} \geq 0$, with the rank $r=\min (m, n)$. The left (right) singular vectors $u_{i}\left(v_{i}\right)$ are the columns of $U(V)$, respectively.

The meaning of the decomposition is the following. For strictly periodic $x(k)$ with period $N, x(k)=x(k+N)$ and $\operatorname{rank}\left(A_{n}\right)=1$ when $n=N$. In this case, $s_{1}>0, s_{2}=s_{3}=$ $\ldots=s_{r}=0 ; s_{1} / s_{2}=\infty$. The vector $v_{1}$ is the (normalised) periodic pattern, the elements of $u_{1} s_{1}$ are the scaling factors; here, they are all equal. For nearly periodic $x(k)$ with period $N$, but $x(k) \neq x(k+N)$, two cases are possible: (1) same repeating pattern, but with different scaling; (2) nearly repeating patterns, with different scaling. In case (1), $\operatorname{rank}\left(A_{n}\right)=1$ for $n=N, s_{1}>0, s_{2}=s_{3}=\ldots=s_{r}=0$, and $s_{1} / s_{2}=\infty$. The vector $v_{1}$ is still the periodic pattern. In case (2), the matrix $A_{n}$ can be full-rank, and $s_{1} \gg s_{2}$.

Summarising, $s_{1} \gg s_{2}$ indicates a strong primary periodic component of the length $n$, given by rows of the matrix $u_{1} s_{1} v_{1}^{T}$. To obtain the further component, one can iterate the above procedure for the residual matrix $A_{n}-u_{1} s_{1} v_{1}^{T}$.

The study [17] defines the periodicity spectrum (P-spectrum) as the variation of the ratio $s_{1} / s_{2}$ with $n$. Considering the dominant maxima of the P-spectrum at $n=N, 2 N, 3 N$, etc., one selects $N$ with the highest maximum count as the most probable value of the period length. (See [17] for technical details.) Following the same basic idea, we consider two alternative measures of signal periodicity

$$
P_{1}=1-\frac{s_{2}}{s_{1}} \quad \text { and } \quad P_{2}=\frac{s_{1}-s_{2}}{s_{1}+s_{2}}, \quad(\text { low }) 0 \leq P_{1}, P_{2} \leq 1 \text { (high) }
$$

and analyse their efficiency on various data. Before doing this, however, we need to introduce some additional steps that render the method applicable to dynamic texture. 


\subsection{Modification for real DT signals}

By a DT signal we mean temporal variation of the average optic flow of a dynamic texture. Figure 5a shows an example of such signal for a low-periodicity DT. This is certainly not the signal one dreams of when working on signal periodicity. The signal rides on a strong nonlinear trend, and its periodicity, if any, may be changing, leading to nonstationarity. The above described basic algorithm is not applicable to such signals, as it cannot cope with varying mean level and non-stationarity: it can only cope with varying amplitude, assuming that scale is largely constant within a segment. These problems should be addressed keeping in mind that SVD is sensitive to shift of signal level.

Three pre-processing algorithms are applied to reduce the effects of noise, signal trend and amplitude variation: (1) the signal is smoothed by a small mean filter to remove noise; (2) the denoised signal is detrended by smoothing it with a large mean filter, then subtracting the result (the mean level) from the denoised signal; (3) the detrended signal is normalised without shifting. Figure $5 \mathrm{~b}$ shows the result of pre-processing the original signal (a). Some periodicity, although low, is now visible.

After the pre-processing, the basic algorithm presented in section 3.1 is applied. However, it is not applied to the entire signal at once. To cope with potential non-stationarity, data windowing is used. A data window slides over the signal, and for each data window position the basic algorithm computes the periodicity defined in eq. (2). To decrease the sensitivity of the method to the starting point within a data window, one can average the result over a range of starting points. In practice, we simply smooth the result of data windowing (periodicity versus window position) by yet another mean filter. As suggested in [17], the size of data window should span at least four periods of the signal.

We use the fast modified Golub-Reinsch algorithm implemented in $\mathrm{C}++$ in the GNU Scientific Library (GSL) available at [13]. The complete processing of a DT signal of length 250 takes less than one second on a $3 \mathrm{GHz}$ machine. This is less than the time needed to compute the signal from a DT sequence of this length by any flow technique.

\subsection{Other possibilities of periodicity estimation}

The study [17] provides arguments and numerous tests in favour of the authors' opinion that, in signal periodicity estimation, SVD is superior to FFT on real quasi-periodic data. Many synthetic and real signals, from very different applications, are processed with both methods and compared to justify the claim.

Without going into details, we can summarise the conclusions of [17] as follows. FFT decomposes a signal into sinusoidal patterns (harmonics) of infinite length. Each pattern has a fixed scale. FFT is likely to miss the prime periodicity when (a) the actual optimal pattern varies from sinusoidal pattern of same length, and/or (b) scaling over successive repetitive segments is different, and/or (c) the noise is strong. The superiority of SVD stems from the following: (a) SVD can cope with any form of the repeating pattern; (b) it can cope with different scaling; and, finally, (c) it provides the most robust rank assessment (nearness to rank one).

Accepting the outcome of the comprehensive study [17], we did not include FFT in our tests. Since it is desirable to compare the SVD method to another approach, we designed and implemented two other, simpler methods based on measuring the distances between signal minima or zero-crossings ( $\mathrm{ZCs}$ ). The rationale behind focusing on the 
dominant minima is that the periodicity of a dynamic texture is often perceived due to the turning points of the optic flow: the flow stops, then moves back.

The minima-based algorithm receives the same pre-processed and normalised signal as the SVD-based one. First, dominant minima are detected as the turning points of the flow. Then, we accumulate the histogram of distances between consecutive minima and remove the outliers using the alpha-trimmed filter that discards the lowest $20 \%$ and the highest $20 \%$ of the distance spectrum. In the remaining distance histogram, the mean is taken as the period. The mean absolute deviation from the period is divided by the period, to serve as a measure of randomness, as opposed to periodicity. To cope with non-stationarity, the same data windowing procedure is used as in the SVD case.

Zero-crossings are closely related to minima. For completeness, they have also been tested. The only difference is that here the histogram accumulates the distances between the second nearest, rather than consecutive, zero-crossings.

\section{Experimental results and conclusions}

Tests with generated signals were carried out in order to (a) compare the two periodicity measures defined in eq. (2), and (b) analyse the robustness of periodicity estimates by SVD, minima and zero-crossings. Examples of synthetic signals generated with additive Gaussian noise are are shown in figure 1. As $\sigma_{\text {noise }}$ approaches 1.0, the noise amplitude exceeds that of the original signal, and periodicity seems to completely disappear. One can observe in figure 2 that this is roughly the point when the period length estimation by SVD becomes unstable and imprecise. At the same time, the periodicity plots demonstrate correct behaviour for both measures. In our further experiments, we use $P_{1}$ because of the more gradual and linear decrease of the periodicity with $\sigma_{\text {noise }}$. However, $P_{2}$ can also be used.
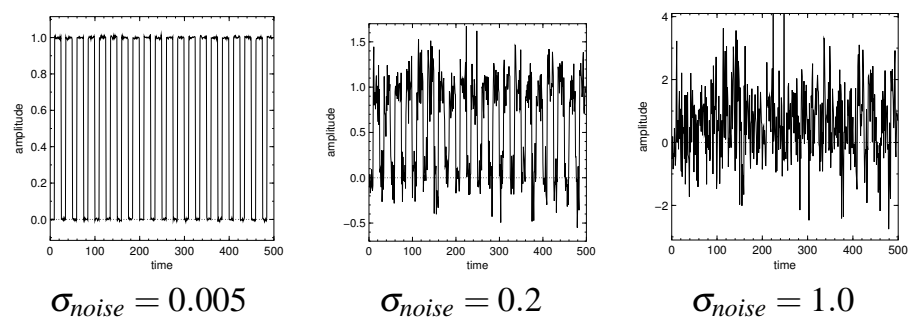

Figure 1: Examples of signals generated with growing amplitude noise. Period is 25.

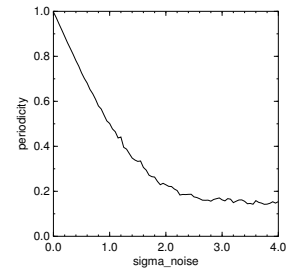

$1-s_{2} / s_{1}$

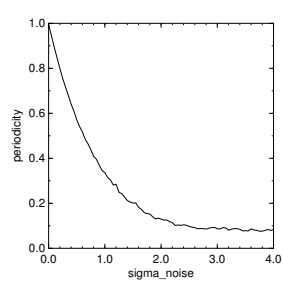

$\left(s_{1}-s_{2}\right) /\left(s_{1}+s_{2}\right)$

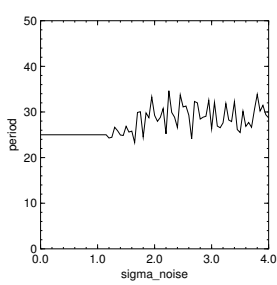

estimated period

Figure 2: SVD estimates of periodicity and period length for growing $\sigma_{\text {noise }}$. 
Figure 3 presents plots of periodicity (i.e., 1-randomness) and period length estimates by the minima algorithm and the $\mathrm{ZC}$ algorithm. The latter yields a more robust period length estimate than the former. (Note that the two $\sigma_{\text {noise }}$ ranges are different!) However, the ZC algorithm yields a very narrow range of periodicity values, which makes it practically unusable for periodicity estimation. In this respect, the minima method is somewhat better. Both minima and ZC are clearly inferior to SVD on this kind of synthetic data.

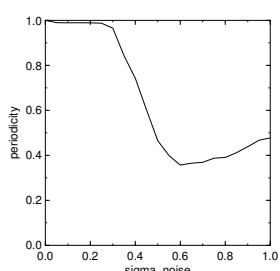

minima periodicity

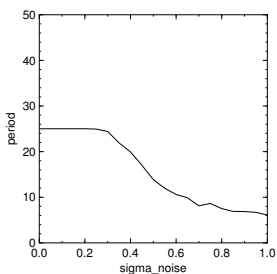

minima period

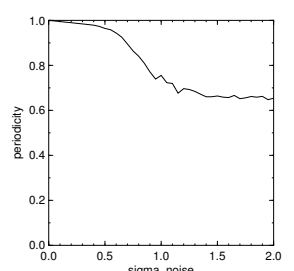

ZC periodicity

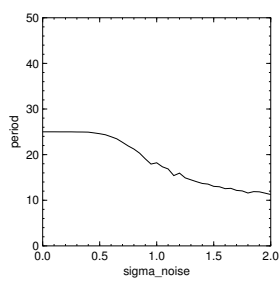

ZC period

Figure 3: Periodicity and period length estimated with minima and zero-crossings.

Tests with real DT signals were done using the DynTex database [23]. Figure 4 shows selected frames of four dynamic textures from the database, while figures 5-8 illustrate the processing of the four DT signals and show the resulting plots of periodicity versus data window position, $P_{1}(k)$. Two periodicity features are computed and presented in table 1: the average periodicity over all data windows $\left\langle P_{1}\right\rangle$ and the relative absolute variation $\delta_{\text {rel }}$. The estimated values are generally consistent with our expectations concerning the character and the ordering of periodicities for the four DTs. In particular, the two views of an escalator differ in periodicity because the close view (a single step) is much more stable. The optic flow in the distant view varies considerably as the white painted step enters the viewfield, grows, then leaves. These variations are reflected by the periodicity plot in figure $7 \mathrm{c}$.

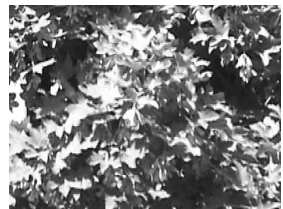

Leaves

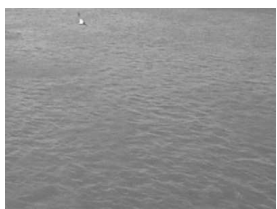

Danube

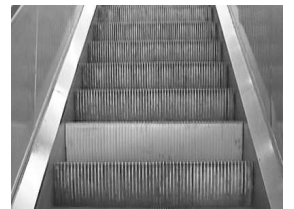

Escalator

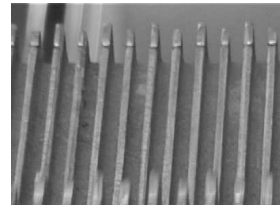

Escalator Step

Figure 4: Selected frames of four dynamic textures from DynTex database.

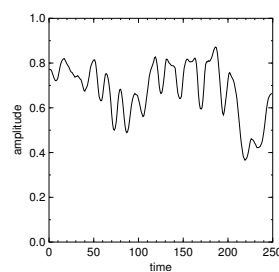

(a) original signal

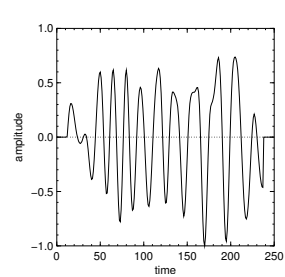

(b) processed signal

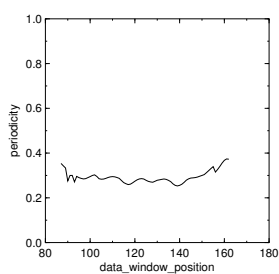

(c) periodicity

Figure 5: Processing dynamic texture Leaves. 


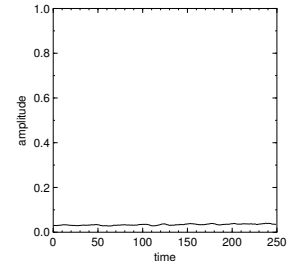

(a) original signal

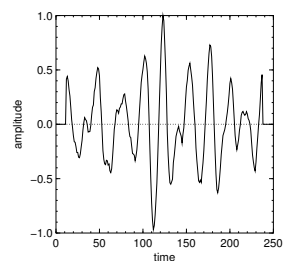

(b) processed signal

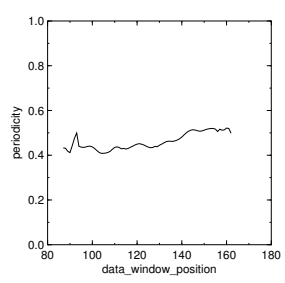

(c) periodicity

Figure 6: Processing dynamic texture Danube.

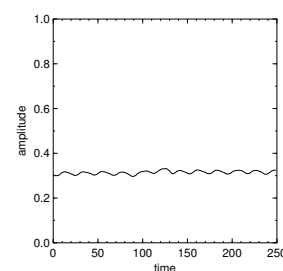

(a) original signal

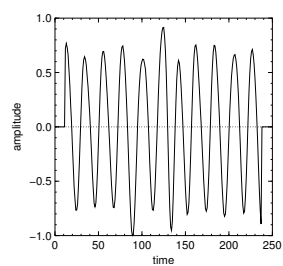

(b) processed signal

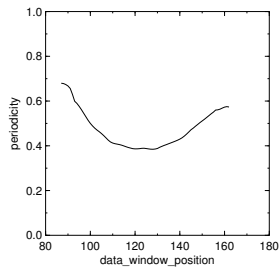

(c) periodicity

Figure 7: Processing dynamic texture Escalator.

Finally, table 2 demonstrates that periodicity can be helpful in dynamic texture classification. The classification test uses the same 26 DTs from the DynTex database and the same optic flow features as the study [12]. However, the experimental protocol is different, as in the current study the sequences are divided in space to obtain samples, while in [12] they are divided in time. (We had to use spatial division to keep signals long enough for periodicity analysis; for this reason, no direct comparison to [12] is possible.) Table 2 shows that classification rate improves consistently when two periodicity features, $\left\langle P_{1}\right\rangle$ and the average minima randomness, are added to the 16 original optic flow features introduced in [12]. In particular, the BBPW optic flow [4] yields the best result when no confidence measure is used; the result improves by almost $4 \%$ when periodicity is added. For the Haussecker confidence measure [14], the gain is almost 4\%. The best classification rate of over $95 \%$ was achieved with periodicity using the CVHS flow $[15,16]$ and the warping error confidence. Of the two periodicity features useful for classification, most of the gain can be attributed to the SVD feature.

To summarise, we conclude that SVD-based estimation of temporal periodicity of dynamic texture is feasible. For successful application, signal pre-processing, normalisation, and data windowing are essential, as many DT signals are non-stationary. Our preliminary experience shows that period length estimation in real data is not sufficiently robust. Concerning DT classification, we observed that the range of DT periodicities in the DynTex database is quite narrow, and the classes may overlap. Our tests show that

\begin{tabular}{lccc}
\hline dynamic texture & periodicity $\left\langle P_{1}\right\rangle$ & variation $\delta_{\text {rel }}$ & characteristic \\
\hline Leaves & 0.293 & 0.067 & low, stable periodicity \\
Danube & 0.461 & 0.069 & mid-range, stable periodicity \\
Escalator & 0.477 & 0.152 & rather high, varying periodicity \\
Escalator Step & 0.716 & 0.033 & high, very stable periodicity \\
\hline
\end{tabular}

Table 1: Average periodicities and relative variations. 


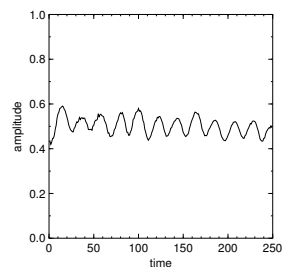

(a) original signal

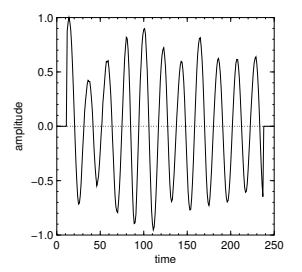

(b) processed signal

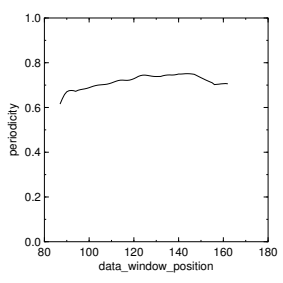

(c) periodicity

Figure 8: Processing dynamic texture Escalator Step.

\begin{tabular}{lccc}
\hline conf. measure & optic flow & without per. & with per. \\
\hline none & BBPW & $79.8 \%$ & $83.7 \%$ \\
Haussecker & BBPW & $85.6 \%$ & $89.4 \%$ \\
warp error & CVHS & $92.3 \%$ & $95.2 \%$ \\
\hline
\end{tabular}

Table 2: Classification results for 26 DTs from DynTex.

SVD periodicity is useful in DT classification, but one should not expect too much. The other two methods for periodicity estimation we tested are less efficient, because both are sensitive to noise; in addition, zero-crossings are too sensitive to signal level variation. SVD may be sensitive to multiple periodic components and to period length variation. Further research is needed on a larger database with longer signals, where more periods can be observed. The method is invariant to rotation and scaling in the image plane. To achieve affine invariance, other flow features should be considered.

Acknowledgments. This work was supported by the EU Network of Excellence MUSCLE (FP6-507752). The authors thank T. Brox, A. Bruhn, N. Papenberg and J. Weickert, as well as T. Amiaz and N. Kyriati, for providing their optic flow programs.

\section{References}

[1] T. Amiaz and N. Kiryati. Dense discontinuous optical flow via contour-based segmentation. In ICIP, volume 3, pages 1264-1267, 2005.

[2] O. Boiman and M. Irani. Detecting Irregularities in Images and in Video. In ICCV, volume 1, pages 462-469, 2005.

[3] P. Bouthemy and R. Fablet. Motion characterization from temporal cooccurrences of local motion-based measures for video indexing. In $I C P R$, volume 1, pages 905908, 1998.

[4] T. Brox, A. Bruhn, N. Papenberg, and J. Weickert. High accuracy optical flow estimation based on a theory for warping. In ECCV, volume 3024 of Springer LNCS, pages 25-36, 2004.

[5] D. Chetverikov. Pattern regularity as a visual key. Image and Vision Computing, 18:975-986, 2000.

[6] D. Chetverikov and R. Péteri. A brief survey of dynamic texture description and recognition. In $4^{\text {th }}$ Int. Conf. on Computer Recognition Systems (CORES'05), Springer Advances in Soft Computing, pages 17-26, 2005. 
[7] G. Doretto, A. Chiuso, S. Soatto, and Y.N. Wu. Dynamic textures. Int. Journal of Computer Vision, 51:91-109, 2003.

[8] I. Laptev et al. Periodic Motion Detection and Segmentation via Approximate Sequence Alignment. In ICCV, volume 1, pages 816-823, 2005.

[9] J.H. Hays et al. Discovering texture regularity via higher-order matching. In ECCV, 2006. To appear.

[10] W. Lin et al. Quantitative evaluation on near regular texture synthesis. In CVPR, 2006. To appear.

[11] R. Fablet and P. Bouthemy. Motion recognition using nonparametric image motion models estimated from temporal and multiscale co-occurrence statistics. IEEE Tr. PAMI, 25:1619-1624, 2003.

[12] S. Fazekas and D. Chetverikov. Normal versus complete flow in dynamic texture recognition: a comparative study. In Texture 2005: $4^{\text {th }}$ Int. Workshop on Texture Analysis and Synthesis, pages 37-42, 2005.

[13] Free Software Foundation, Inc. GNU Scientific Library (GSL), version gsl-1.7. http://www.gnu.org/software/gsl/, 2005.

[14] H. Haussecker, H. Spies, and B. Jähne. Tensor-based image sequence processing techniques for the study of dynamical processes. In Int. Symp. On Real-time Imaging and Dynamic Analysis, pages 704-711, 1998.

[15] B. Horn and B. Schunck. Determining optical flow. Artificial Intelligence, 17:185203, 1981.

[16] Intel Corporation, Microprocessor Research Labs. OpenCV: Open Source Computer Vision Library. www. intel . com/technology/computing/opencv/, 2005.

[17] P.P Kanjilal, J. Bhattacharya, and G. Saha. Robust method for periodicity detection and characterization of irregular cyclical series in terms of embedded periodic components. Physical Review E, 59:4013-4025, 1999.

[18] F. Liu and R.W. Picard. Periodicity, Directionality, and Randomness: Wold Features for Image Modeling and Retrieval. IEEE Tr. PAMI, 18:722-733, 1996.

[19] Z. Lu, W. Xie, J. Pei, and J. Huang. Dynamic texture recognition by spatio-temporal multiresolution histogram. In IEEE Workshop on Motion and Video Computing (WACV/MOTION'05), 2005.

[20] R.C. Nelson and R. Polana. Qualitative recognition of motion using temporal texture. CVGIP: Image Understanding, 56:78-89, 1992.

[21] C.H. Peh and L.-F. Cheong. Exploring video content in extended spatio-temporal textures. In 1st European workshop on Content-Based Multimedia Indexing, pages 147-153, Toulouse, France, 1999.

[22] C.H. Peh and L.-F. Cheong. Synergizing spatial and temporal texture. IEEE Tr. on Image Processing, 11:1179-1191, 2002.

[23] R. Péteri, M. Huskies, and S. Fazekas. DynTex: A comprehensive database of Dynamic Textures. www.cwi.nl/projects/dyntex/, 2005.

[24] B. Sarel and M. Irani. Separating Transparent Layers of Repetitive Dynamic Behaviors. In ICCV, volume 1, pages 26-32, 2005.

[25] J.R. Smith, C.-Y. Lin, and M. Naphade. Video texture indexing using spatiotemporal wavelets. In ICIP, volume 2, pages 437-440, 2002. 